\title{
A Simple and Standardized Method for Analysing Head and Face Morphology of a Population Sample
}

\author{
Yohann KELKEL ${ }^{* a}$, Matthieu FOISSAC ${ }^{b}$, Laurent BALY ${ }^{c}$ \\ ${ }^{a}$ R\&D engineer, Movement Science, Oxylane research, Villeneuve d'Ascq (59), France \\ bLaboratory Manager, Movement Science, Oxylane research, Villeneuve d'Ascq (59), France \\ ${ }^{c} R \& D$ Director, Oxylane research, Villeneuve d'Ascq (59), France
}

\begin{abstract}
In order to design products that will interact with head and face, engineers need to build a solid knowledge about their target customer's morphology. Classical methods allow only a definition of different size groups derived from specific 2D anthropometric measurements such as head girth, bizygoma-tic breadth, mouth breadth ... (Snyder 1978 [2]). Acquiring 3D geometry is another method that is being used increasingly for the past 20 years. From Bradtmiller in 1993 [3] to Mochimaru in 2008 [8], 3D scans offer the possibility to carry a full analysis of morphological details. Indeed, using 3D point clouds instead of classical 2D anthropometric measurements allows taking into account the curvature variations in specific areas such as cheek or chin. Some methods are using mathematical functions (Biasotti 2008 [7]), others are working with an analogous 3D model associated with anatomical landmarks (Mochimaru 2008 [8] ; Ma 2005 [6]). Most of those methods are trying to classify population into size groups which are based on global 3D variations such as depth, width and height of the model. Those groups are then represented with average 3D model (Kouchi 2004 [5]).
\end{abstract}

Our goal here is to compute a simple and standardized method for analysing head and face morphology. It had to give results that directly take into account more details than just global dimension variations through an average $3 \mathrm{D}$ model and that could be directly usable to validate the right interface geometry of a computer aided designed product.

The sample population was composed of 51,15 to 47 year old women and 64,16 to 55 year old men. The digitalisation was performed using a Konica Minolta VI-910 laser scanner. Every subject's face was digitalized using three automatically registered views. The reconstructed scanned faces were then oriented along a reference plane computed from 3 anatomical landmarks (Projection of the Nasion on the face's symmetry plane, Mental protuberance and Exocanthion). The same plane was then used to extract parallel level curves of the face (Figure 1). After normalizing all the curves in polar coordinates, every mean level curve was calculated on our scanned population.

Using the whole population, we manage to compute a mean 3D face mapped with the geometric standard deviation (STD) (Figure 8). This preliminary result is a first step toward an easy face / product interface design for manufacturers as it provide them with the zone of less significant morphology disparity (The green zones).

However, the use of a common reference point was necessary to register every face of our sample, and moving this point to another location changed the global standard deviation mapping - which is easily explained by a null STD at that point -. The anatomical landmark picking required (in this method) are also a source of inaccuracy. In order to avoid those two main limits, further work is necessary to use an automatic registration and spherical normalisation (allowing not working with a reference point and landmarks). Finally, this method should be validated in regard to traditional anthropometric methods (2D measurements).

Keywords: $3 d$ body scanning, proceedings, format

*yohann.kelkel@oxylane.com; +33 (0)359318811 


\section{Introduction}

There are several possibilities when it comes to designing or improving products that will have a strong interaction with the body. The most efficient is without a doubt the "made to measure" solution that would take into account every personal measurement to design the product. This solution appears to take a lot of development time as well as to rise design and production costs. Therefore, when designing a product, it is more cost and time effective to consider Mass customization. This last solution demands a certain knowledge of the body form of our targeted population sample in order to design a product that will fit everyone properly.

There are several methods we could use to measure body dimensions, and each method uses specific tools. Before the 90's, tapes and calipers allowed to take two dimensional measurements from sample populations. These measurements were then statistically analyzed to then design products based on clusters and percentiles.

Biasoti et all [7], are exposing the fact that statistical analysis of 2D measurements are usually not usable when it comes to designing a product that has to follow 3D curves of the body. Moreover, Howells [1] found for example that there is no correlation between head width and head depth. It is difficult indeed to find the right correlations between 2D measurements that would enable best fit of the product. Therefore, in order to improve the fitting, it is necessary to take as many measurements as possible and, the best way to do so without losing precision and time is 3D scanning.

In 1993, Bradtmiller [3] used a 3D scanner to digitalize 475 females and 462 males' head in order to design helmets. He justifies the use of 3D measurement with the poor correlation between head measurements such as head circumference and head height. His method consists in statistical analysis of vectors extracted from curves parallel to the sagittal plane and a reference point.

In 2006 Ma uses a spherical decomposition of 3D models. He then analyzes its sample using an extended Gaussian image analysis.

Finally, In order to design well fitted spectacle frames, Mochimaru and Kouchi $[5,8]$ are analyzing homologous head decomposition of 52 and 56 males through distance matrices and Multidimensional scaling (MDS). They also are the only ones to use physical markers and classical 2D anthropometric measurement (These measurements were used to define the principal variations of their MDS)

Concerning 3D analysis, interesting methods are also used in the field of face or more generally form recognition. LAI et al [4] for example are using a reduced 3D model based on cylindrical coordinates. They are then analyzing 2D surface function of the 3D human heads to compute a mean head and the principal 3D head shapes. In order to register every 3D head for its analysis, Lai uses a reference surface. Another method can be found in Biasoti's article [7] where he works with mathematical "size function" after decomposing the 3D form as level lines along its principal axis.

When considering 3D face analysis, we wanted to use a simple method that would enable us to reduce the calculation time and to provide engineers and designers with a comprehensive model on which their work could be based. This paper relates the first step in defining this new method to analyze head and face morphology.

\section{Material and methods}

\subsection{Subjects}

Subjects were 64 adult male volunteers of $30 \pm 8$ years (from 16 to 55 years old) and 51 females of 27 \pm 7 years (from 15 to 47 years old). Figure 1 illustrates subject distribution of our sample population. Note that this population was not chosen based on a specific sampling strategy as it was used to create and validate our method and not to design a specific product. 


\subsection{Material}

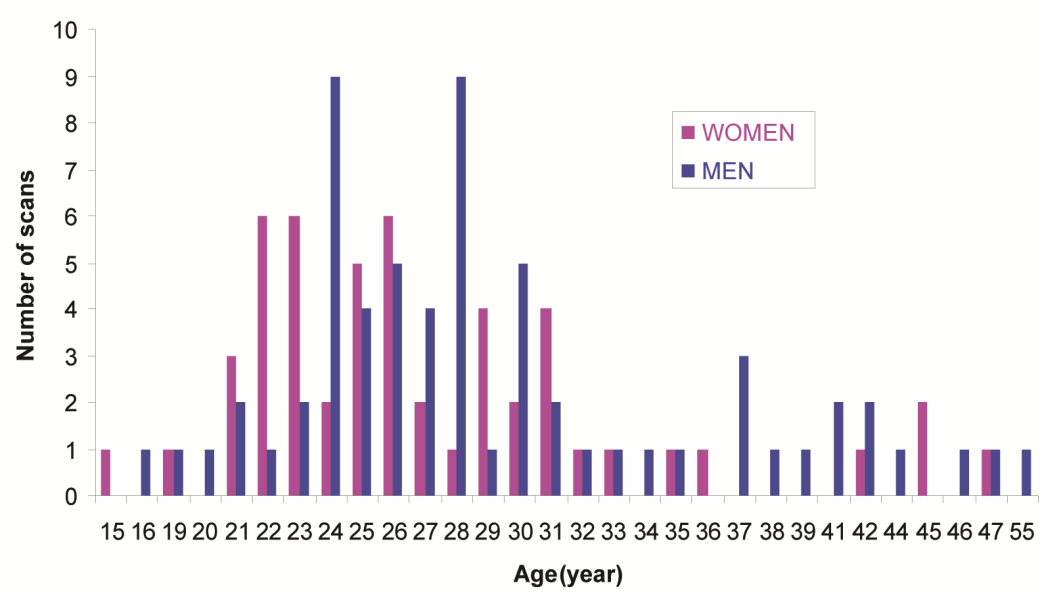

Fig. 1. Subjects' age and sex distribution

At the time of the survey, the best identified 3D digitalizer was the Konica Minolta scanner, vivid910. Its integrated calculation software enabled us to reconstruct faces from 3 views in a limited amount of time. Moreover compared to previously tested technology this one was the fastest in acquiring one view $(0,3$ s) with a good precision (less than $2 \mathrm{~mm}$ in the 3 dimensions)

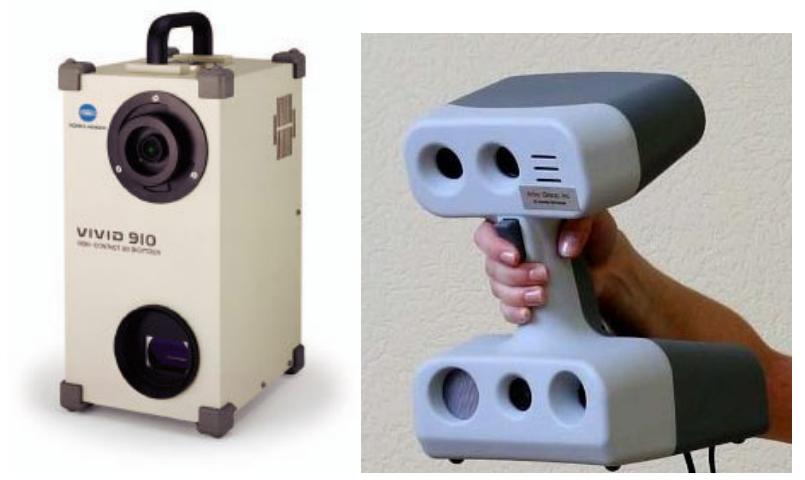

Fig. 2. Vivid 910 non contact 3D digitalizer from Konica Minolta (Left) MH-Walle 3D scanner from ARTEC3D (right) For the next steps of this study we used an even fastest technology enabling us to digitalize quickly full heads (in less than a minute). This optic solution is using white light pattern instead of laser, capturing 11 views per seconds. With this new solution (Scanner MH-Walle from ARTEC3D) we cover an error of less than $2 \%$ on any measurement taken on the 3D model.

As the analysis method we want to develop is bound to be used in an industrial design process, we wanted to identify the best technology for digitalizing specific body parts with a good precision and as quickly as possible. We were able to use the second solution to scan 1500 men and women over 3 month around Europe. These data are currently being processed to validate and improve the method presented in this paper.

All the 3D files manipulation has been made using Rapidform or Geomagic software. The analysis and automation of the extracted curves was computed with Matlab.

\subsection{Protocol}

Every subject is seated in front of the scanner on a chair that can pivot at $360^{\circ}$. The scanner is placed under the subject (as illustrated on Figure 3) and facing towards the top so as to capture as much of the subject chin as possible. We asked the subject to turn his whole chair (and therefore not to change his position) and to keep the same facial expression during the capture of the 3 views. Subjects have to look left, face and right of the scanner, and each captured view is directly registered using the Konica Minolta PET software ( 3 point registration with least square refinement calculation between surfaces). If the subject had long hair, he was asked to attach it at the top back of the head and to put all remaining hair behind its ears. 


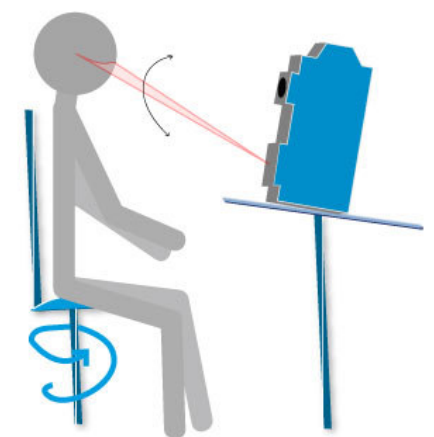

Fig. 3. Illustration of the measurement protocol

\section{Analysis method}

Before compiling and comparing our 115 digitalized faces, we need to "normalize" point clouds. From the different methods used in the literature we wanted to use a method similar to Bradtmiller [3] as we found it would be the most adapted to the design of a product. Working with a reference plane would indeed allow us to chose it according to the product we are willing to design.

\subsection{Reference frame}

Figure 4 illustrate the placement of our virtual landmarks (We call them virtual because there is no previous physical marking on the subject before the scanning process). Those 6 landmarks are defined as: $A=$ Nasion; $B=$ Pronasale point; $C=$ Stomion; $D=$ Pogonion; $E=$ Exocanthion ; $\mathrm{F}=$ Projection of the point $\mathrm{E}$ in the calculated plan of symmetry (S Plane). The symmetry plane is automatically calculated with CAD software such as Rapidform or Geomagic. It is logical here to work with symmetries as we will be aiming to design a symmetrical product.

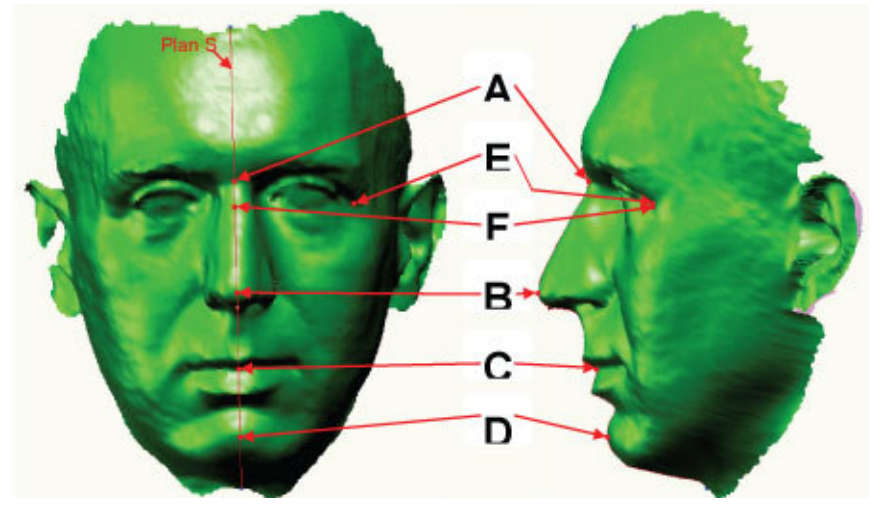

Fig. 4. Placement of the 6 reference points

The reference frame $R_{0}(1)$ is computed using the previously defined virtual landmarks (the three vectors are detailed in (2)).

$$
\vec{x}=\frac{\overrightarrow{D E}}{\|\overrightarrow{D E}\|} \quad \vec{z}=\frac{\{\overrightarrow{D E} \wedge \vec{x}, \vec{y}, \vec{z}\}}{\|\overrightarrow{D E} \wedge \overrightarrow{D F}\|} \quad \vec{y}=\vec{x} \wedge \vec{z}
$$

The reference plane is passing through points $D, E$ and $F$.

\subsection{Defining level lines}

Level lines are defined as every section parallel to the previously computed reference plane. Each section plane is then spaced of a distance $\delta$ defined in formula (3):

$$
\vec{\delta}=\delta \cdot \vec{z}
$$

Figure 5 shows an example scan with extracted lines parallel to the reference plane defined in 3.1. In order to allow more or less precision in our calculation, we can choose the distance $\delta$ between each extracted curve (In Figure 5 we have chosen $\delta=1 \mathrm{~mm}$ ). 


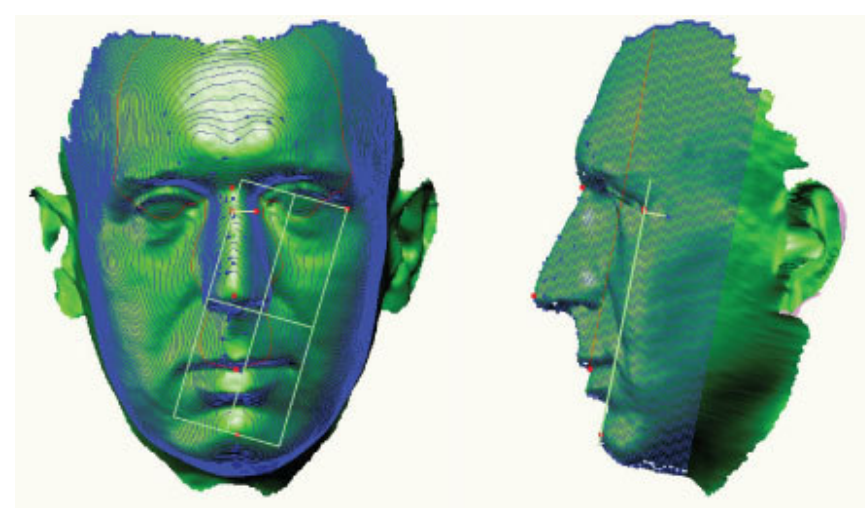

Fig. 5. Model with extracted lines $(\delta=1 \mathrm{~mm})$

As the scanned face doesn't have clean boundaries, prior to curve extraction we had to go thought a cleaning process of every non significant curves. Each line is then exported as a point cloud of approximately 50,000 points.

\subsection{Normalizing level lines}

The first thing to do before normalizing our scans is to reorient them. We did not choose classical registration as it require too much calculation time, therefore we used the reference frame defined in 3.1 to rotate each scan. A simple 3D transformation is realized around each axis:

Around the $z$ axis at an $A z$ angle with:

$$
A z=\arctan \left(\frac{\vec{x}(2)_{R o}}{\vec{x}(1)_{R o}}\right) \quad R z=\left[\begin{array}{ccc}
\cos (-A z) & -\sin (A z) & 0 \\
\sin (-A z) & \cos (A z) & 0 \\
0 & 0 & 1
\end{array}\right]
$$

Therefore we define $\vec{x}^{\prime} \vec{y}^{\prime}$ and $\vec{z}^{\prime}$, the rotation of $\vec{x}, \vec{y}$ and $\vec{z}$ around $z$ of an angle Az.

Around the $y$ axis at an Ay angle with:

$$
A y=\arctan \left(\frac{\vec{x}^{\prime}(3)_{R o}}{\vec{x}^{\prime}(1)_{R o}}\right) \quad R y=\left[\begin{array}{ccc}
\cos (A y) & 0 & \sin (A y) \\
0 & 1 & 0 \\
-\sin (A y) & 0 & \cos (A y)
\end{array}\right]
$$

Therefore we define $\vec{x}^{\prime \prime}, \vec{y}^{\prime \prime}$ and $\vec{z}^{\prime \prime}$, the rotation of $\vec{x}^{\prime}, \vec{y}^{\prime}$ and $\vec{z}^{\prime}$ around $z$ of an angle Ay.

Around the $x$ axis at an Ax angle with:

$$
A x=\arctan \left(\frac{\vec{z}^{\prime \prime}(2)_{R o}}{\vec{z}^{\prime \prime}(3)_{R o}}\right) \quad R x=\left[\begin{array}{ccc}
1 & 0 & 0 \\
0 & \cos (A x) & -\sin (A x) \\
0 & \sin (A x) & \cos (A x)
\end{array}\right]
$$

Now that the scans are oriented correctly we finally compute a translation to a reference point chosen depending of the final product we aim to design. For this example we have chosen point $D$ as the "origin" or reference point. Each scan is therefore translated along the vector defined in (7):

$$
\overrightarrow{O D}_{R o}
$$



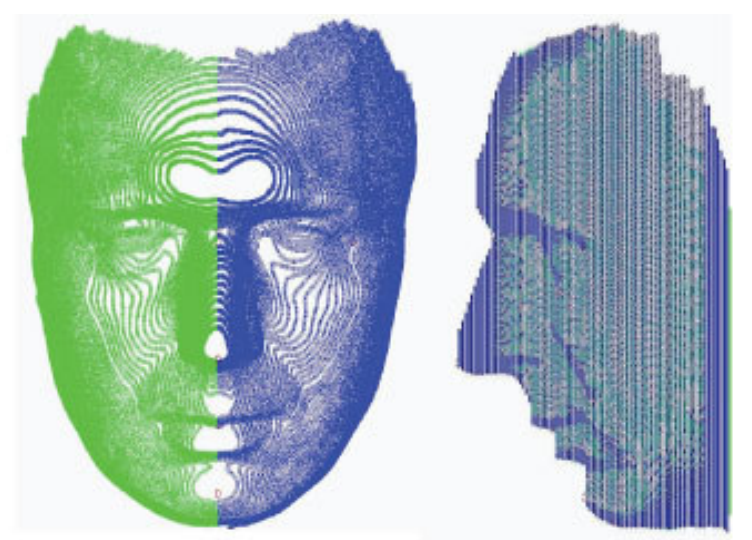

Fig. 6. Rotated and translated scan after level lines extraction

After the scan transformation we had to create homologous models so as to compare them between each other. To do so each curve were normalized in polar coordinate. We extrapolated a point every degree as illustrated on Figure 7. Finally the last step is a simple calculation of the mean curve between right and left side.

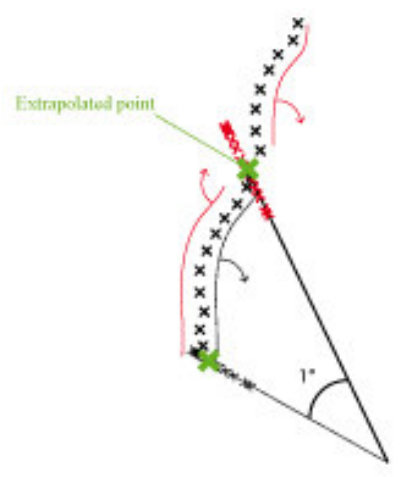

Fig. 7. Normalization method

After creating this homologous model for each scan we were able to compute a simple analysis described in the next part.

\subsection{Analyzing level lines}

In order to offer to our engineers and designer the simplest tool possible to work with, we computed a mean face of our sample. This mean faced is color mapped with the standard deviation found on our sample. (Figure 8)

\section{Results}
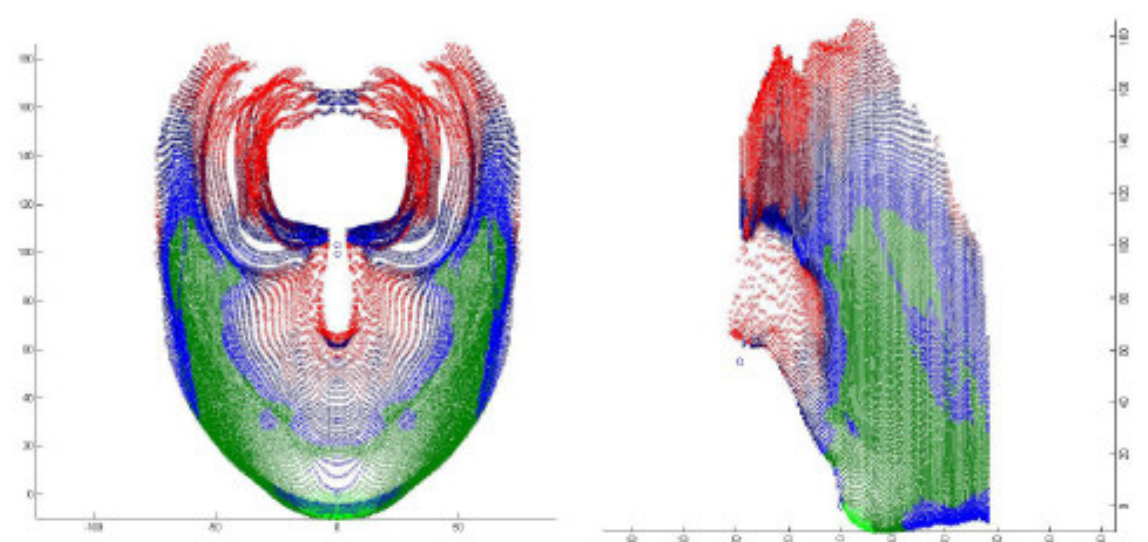

Fig. 8. Results on our 115 people sample $/$ Light green $=S T D<2 m m /$ Deep green $=2 m m<S T D<4 m m /$ Light blue $=4 m m<$ $S T D<6 \mathrm{~mm} /$ Deep blue $=6 \mathrm{~mm}<S T D<8 \mathrm{~mm} /$ Deep red $=8 \mathrm{~mm}<S T D<10 \mathrm{~mm} /$ Light red $=S T D>10 \mathrm{~mm}$ 
Figure 8 represents the mean faces composed of 119 slices of our 115 scanned subjects. The color mapping gives standard deviation on the sample, from green (for a small STD) to red (High STD). This 3D map can now be used to make decisions on how a product interacting with the face is going to be designed and/or how an existing product is going to have to adapt itself (in terms of materials) if it goes through high STD zones.

We can see here that from the chin to cheekbones and going through the cheeks the STD is less than $4 \mathrm{~mm}$. This zone of lesser variability would then be the one to use when having a product interact with the face. Zones with a STD higher than $8 \mathrm{~mm}$ are located around the nose and forehead. Those would be zones we should not consider when design a product.

\section{Discussion}

We can clearly see two main limits to this method. First of all, our registration process is using 3 angles and a reference point to bring models on an analogous base. We know that changing the reference point, would induce changes in the color mapping. This is easily explained by a null STD at the reference point. Further work is therefore needed on the registration process prior to normalisation and comparison between scans. For our next steps, we will be going toward a multiple point registration rather that just one point, therefore minimizing our mapping error.

Our second limit here is the required anatomical landmark picking. This is also a source of inaccuracy as it necessitates a subjective placement of points by an operator.

In order to avoid those two main limits, further work is necessary to use an automatic registration and spherical normalisation (allowing us not to work with a reference point and landmarks).

Finally, our method will have to be validated in regard to traditional anthropometric methods (2D measurements). This validation will be done on a more representative sample (1500 people). We will compare measurement on our mean face and standard 2D anthropometric measurements (and there standard deviation) such as bizygomatic breadth or Nose breadth.

Complementary to this result, we could compute principal component analysis or multidimensional scaling analysis of every slice for the whole population sample. With such computation Mochimaru [8] was able to categorize its population along two dimensions. Doing so would enable us to compute clusters among our sample.

\section{Conclusion}

This paper shows a new method to analyses specific body form of a sample population. Centered on face/head analysis, we managed to come up with a simple method, giving engineers and designers a tool to design product interacting with the face. The computed method hasn't been validated yet and current work is being done on an already scanned (Full head scans) sample of 1500 persons in France, Spain and Italia. In the process of improving our method, we have to keep in mind that our solution has to be time and cost effective as it is meant to be used in product design.

$3 \mathrm{D}$ form analysis is the next step in anthropometry. It takes population morphology analysis to a higher level of precision and allows engineers and designers to come up with products that are always better fitted. The challenge here is to find a way to process a huge amount of data given by 3D scanners into a comprehensive material for everyone that is willing to design human interfaced products.

\section{References}

1. Howells,W.W., (1973): "A study by multivariate analysis of patterns of difference among recent human population", Paper of the Peabody Museum of Archaeology and Ethnology, Harvard University, 67, 259 pp

2. Snyder,R.G, Schneider L.W., Owings,C.L. (1978), "Infant child and teenager anthropometry for product safety design", Advances in Consumer Research Volume 05, eds. Kent Hunt, Ann Abor : Association for Consumer Research, Pages: 499-507.

3. Bradtmiller,B., (1993): "An approach to creating three-dimensional head forms for helmet sizing design", Proceedings of the 31st Annual Symposium of Safe Association, 244-249

4. LAI,S-H , CHEN,Y-L, (2003): "Learning a statistical 3D geometric head model", Proceedings of SPIE-IS\&T Electronic Imaging, SPIE Vol. 5013.

5. KOUCHI,M.,MOCHIMARU,M., (2004): "Analysis of 3D face forms for proper sizing and CAD of spectacle frames", Ergonomics, 1499-1516,(18)

6. Bo,M., Wong,H-S., (2005): "2D clustering based discriminant analysis for 3D head model classification", Pattern recognition, 491-494

7. Biasotti,S., Giorgi, D., Spagnuolo, M., Falcidieno, B., (2008): "Size functions for comparing 3D models", Pattern recognition, 2844-2873, (41)

8. MOCHIMARU,M., KOUCHI,M., (2008): "Technologies for the design and retail service of well-fitting eyeglass frames: - Toward the mass customization business - “, Synthesiology English edition, 1, 38-46 